\title{
MEDIDA DAS CAPACIDADES PSICOMOTORAS ENVOLVIDAS NA TÉCNICA DA INJEÇĀO INTRAMUSCULAR
}

\author{
Ana Maria Kazue Miyadahira *1 \\ Maria Sumie Koizumi **2
}

MTYADAHIRA, A. M. K.; KOIZUMI, M. S. Medida das capacidadeo poicomotoras envolvidas na tócnica da injeçào intramuscular. Rev. Esc. Enf. USP., v. 27, n. 2, p. 263.80, ago. 1993.

Partindo-se do presauposto de que no estudo de habilidades psicomotoras, a medida das capacidades motoras é fundamental, apresenta-se dois testes passiveis de serem utilizados pela enfermagem. Estes testes, denominados "psicomotor eletrônico" $e$ "perfuragem" foram aplicados na primeira etapa do estudo sobre o processo de ensino-aprendizagem de habilidades psicomotoras no qual analisou-se a técnica de injeçào intramuscular. Três grupos de estudantes de graduaçäo em enfermagem, em diferentes estágios de desenvolvimento no curso, foram avaliados e verificou-se que eles eram homogêneos em relaçào às capacidades psicomotoras testadas.

UNITERMOS: Injeçöes in tramusculares. Habilidades psicomotoras.

\section{1 - INTRODUÇÃO}

Partindo da premissa que, para o desenvolvimento de uma habilidade psicomotora, é imprescindível que o indivíduo tenha as capacidades requeridas para esta habilidade, a determinaçāo das mesmas é de suma importância.

A prática da enfermagem, pressupõe competência em uma série de habilidades predominantemente psicomotoras (ALMEIDA ${ }^{1}$, BUTTERFIELD ${ }^{2}$, CARVALHO ${ }^{4}$, ELLIOT et $\mathrm{al}^{5}$, FRIEDLANDER et $\mathrm{a}^{9}$, GOMEZ; GOMEZ ${ }^{10}$, HARDY ${ }^{11}$, INFANTE ${ }^{13}$, MILDE ${ }^{20}$, MOGAN; THORNE ${ }^{21}$, SHEAHAN ${ }^{26}$, SWEENEY; REGAN ${ }^{27}$,SWEENEY et al ${ }^{28}$, WHYTE ${ }^{30}$ ). Essas habilidades compreendem desde as mais simples

1 Professor Doutor do Departamento de Enfermagem Médico-Cirúrgica da Escola de Enfermagem da Universıdade de Sảo Paulo.

2 Professor Associado do Departamento de Enfermagem Médico-Cirúraca da Escola de Enfermagem da Universidade de Sáo Paulo. 
atividades até as mais complexas, as quais envolvem grande número de movimentos coordenados e de alta precisão.

Considerando que a habilidade psicomotora é um dos instrumentos básicos da enfermagem, conclui-se que é indispensável o seu desenvolvimento como um meio de trabalho e não como um fim (HORTA et al ${ }^{12}$, PAIM ${ }^{23}$ ).

O desenvolvimento da habilidade psicomotora especifica se faz através do ensino das técnicas de enfermagem. Em vista disso, os cuidados de enfermagem que exigem habilidade psicomotora no processo ensino-aprendizagem são ministrados de maneira gradativa, em várias etapas, segundo o grau de complexidade para facilitar a aprendizagem.

Um fator relevante que deve ser considerado é que as pessoas diferem na capacidade de aprender habilidades psicomotoras. Estas diferenças sāo devidas a fatores genéticos ou podem ser atribuídas à totalidade de experiências anteriores de cada estudante?

MAGILL ${ }^{17}$ define capacidade motora como um traço geral ou qualidade de uma pessoa, portanto, algo inato relativo a desempenho motor. É um componente da estrutura de uma habilidade motora. Segundo FLEISHMAN ${ }^{7}$, existem onze capacidades perceptivo-motoras identificáveis e mensuráveis e nove capacidades designadas pelo autor de capacidades de proficiência física. Estas últimas diferem das perceptivo-motoras por serem mais comumente ligadas ao desempenho atlético e físico geral. Já a habilidade psicomotora compreende atos ou tarefas que requerem movimento e devem ser aprendidos a fim de serem executados corretamente.

No aprendizado de uma habilidade, predominantemente psicomotora, quais as capacidades subjacentes requeridas para uma execução correta da mesma?

Assim, quando se fala em aprendizagem da habilidade da técnica da injeção intramuscular (IM), pressupooe-se a existência de uma série de capacidades básicas envolvidas nesta atividade.

Em 1972, FLEISHMAN ${ }^{8}$ apresentou o desenvolvimento de uma "taxionomia" de capacidades perceptivo-motoras humanas. Neste estudo, o autor identificou e mensurou onze capacidades perceptivo-motoras além das nove capacidades de proficiência física. $\mathrm{Na}$ técnica da IM, algumas dessas capacidades perceptivo-motoras estão presentes, porém, nenhuma atividade de proficiència física é necessária, pois elas sāo mais comumente ligadas ao desempenho atlético e físico geral.

$\mathrm{Na}$ execução da técnica da IM (FLEISHMAN ${ }^{\gamma}$ ), pode-se identificar as seguintes capacidades perceptivo-motoras:

- "coordenação multimembro" - a capacidade de coordenar o movimento dos membros simultaneamente; 
- "precisāo de controle" - a capacidade de executar ajustes musculares altamente controlados e precisos em que grupos maiores de músculos estāo envolvidos;

- "velocidade do movimento do braço" - a capacidade de fazer um movimento geral e rápido do braço;

- "controle de graduação" - a capacidade de mudar a velocidade e a direção de respostas no tempo adequado;

- "destreza manual" - a capacidade de fazer movimentos de braço e mäos hábeis, bem direcionados;

- "destreza dos dedos" - a capacidade de executar movimentos controlados, hábeis de objetos pequenos, envolvendo principalmente os dedos;

- "estabilidade braço-māo" - a capacidade de fazer movimentos precisos de posicionamento de braços e mäos nos quais a força e a velocidade têm um envolvimento mínimo;

- "pontaria" - a capacidade de apontar com precisāo para um objeto pequeno no espaço.

Estas capacidades, corresponderiam à coordenaçāo motora, prontidão motora e memória imediata, necessárias para a execuçāo das técnicas de enfermagem, como a deste estudo.

Em relação à habilidade psicomotora da técnica da IM, considerar-se-á a classificaçāo por sistemas descritos por MAGILL ${ }^{16}$ e composto por: (1) precisāo do movimento; (2) caráter bem defínido dos pontos iniciais e finais; (3) estabilidade do meio a mbiente e (4) controle por "feedback" (retroinformação).

1. Precisão do movimento - com base na precisão do movimento envolvido na habilidade, a técnica da IM classifica-se basicamente dentro da categoria de "habilidade motora fina", pois esta técnica requer controle de músculos pequenos do corpo a fim de atingir a execução bem sucedida desta habilidade. SAGE ${ }^{24}$ classifica a habilidade motora como fina ou global conforme o tamanho do músculo envolvido, a quantidade de força aplicada e a amplitude do movimento. Esta habilidade envolve coordenação óculo-manual e requer um alto grau de precisão no movimento para o desempenho da habilidade específica, num nível elevado de realizaçāo. Embora a técnica da IM seja classificada na categoria de "habilidade motora fina", dependendo da situação, como por exemplo, do local que o paciente vai receber a injeção IM e o seu posicionamento, pode haver o envolvimento da grande musculatura como base de sustentação daqueles movimentos. Portanto, ambas as categorias de habilidades nāo podem ser consideradas mutuamente exclusivas, como "globais" versus "finas".

2. Pontos iniciais e finais bem definidos - dentro desta classificação a técnica da IM é considerada como uma "habilidade motora 
discreta serial", pois tem pontos iniciais e finais bem definidos como os passos e os princípios da técnica, é executada em uma ordem definida e em seqüência. Neste tipo de habilidade serial, cada parte ou fase da técnica como serrar, limpar, quebrar e aspirar a medicação é um estímulo e uma resposta ao movimento imediatamente anterior e um estímulo para o movimento seguinte.

3. Estabilidade do a mbiente - a técnica da IM é classificada como uma "habilidade fechada", por ocorrer em condiçōes fixas sem mudanças do a mbiente e se tratar de "tarefa autocompassada", pois o executante da habilidade é que determina quando e como iniciar a técnica; não é determinada por uma fonte externa, o estímulo.

4. Controle de "feedback" (retroinformaçäo) - considerando que este sistema de classificaçāo está baseado na forma e no momento do retorno de uma informação sensorial, podendo ser usado para controlar o movimento, a técnica da IM é classificada como uma "habilidade de circuito fechado", pois a informaçāo pode ser usada para se fazer alguns ajustes antes que o movimento seja completado. Assim, no movimento de aplicar a IM, se se percebe que a introdução da agulha não irá formar um ângulo de $90^{\circ}$, pode-se alterar a posição da mão, na tentativa de se ajustar a angulação.

Assim, conhecer instrumentos que viabilizem a avaliaçāo das capacidades envolvidas em determinada habilidade psicomotora é fundamental para o processo ensino-aprendizagem da mesma.

Diante do exposto, neste estudo temos como objetivo:

Avaliar a capacidade psicomotora dos estudantes do curso de Graduação em Enfermagem, através de testes instrumentais ("psicomotor eletrônico" e "perfuragem").

\section{2 - METODOLOGIA}

A população foi constituída de 141 estudantes do curso de Graduaçāo em Enfermagem da Universidade de Sāo Paulo, divididos em três grupos:

Grupo A - 47 estudantes do $3^{\circ}$ semestre;

Grupo B - 47 estudantes do $4^{\circ}$ semestre;

Grupo C -47 estudantes do $8^{\circledR}$ semestre.

Foram aplicados dois testes instrumentais, denominados pelas autoras deste estudo como:

. "Teste Psicomotor Eletrônico" (medidor C.C.N. TESTER modelo C.P. - C.M.) e

- "Teste de Perfuragem". 


\section{A. DESCRIÇÃO DO TESTE PSICOMOTOR ELETRONICO}

A prova com o aparelho eletrônico C.C.N modelo CP.CM (figuras 1 e 2) foi utilizada com o objetivo de avaliar algumas capacidades perceptivo-motoras, necessárias para o desenvolvimento da habilidade psicomotora da técnica da IM, que envolve basicamente movimentos que exigem memória imediata, coordenação e prontidão motora.

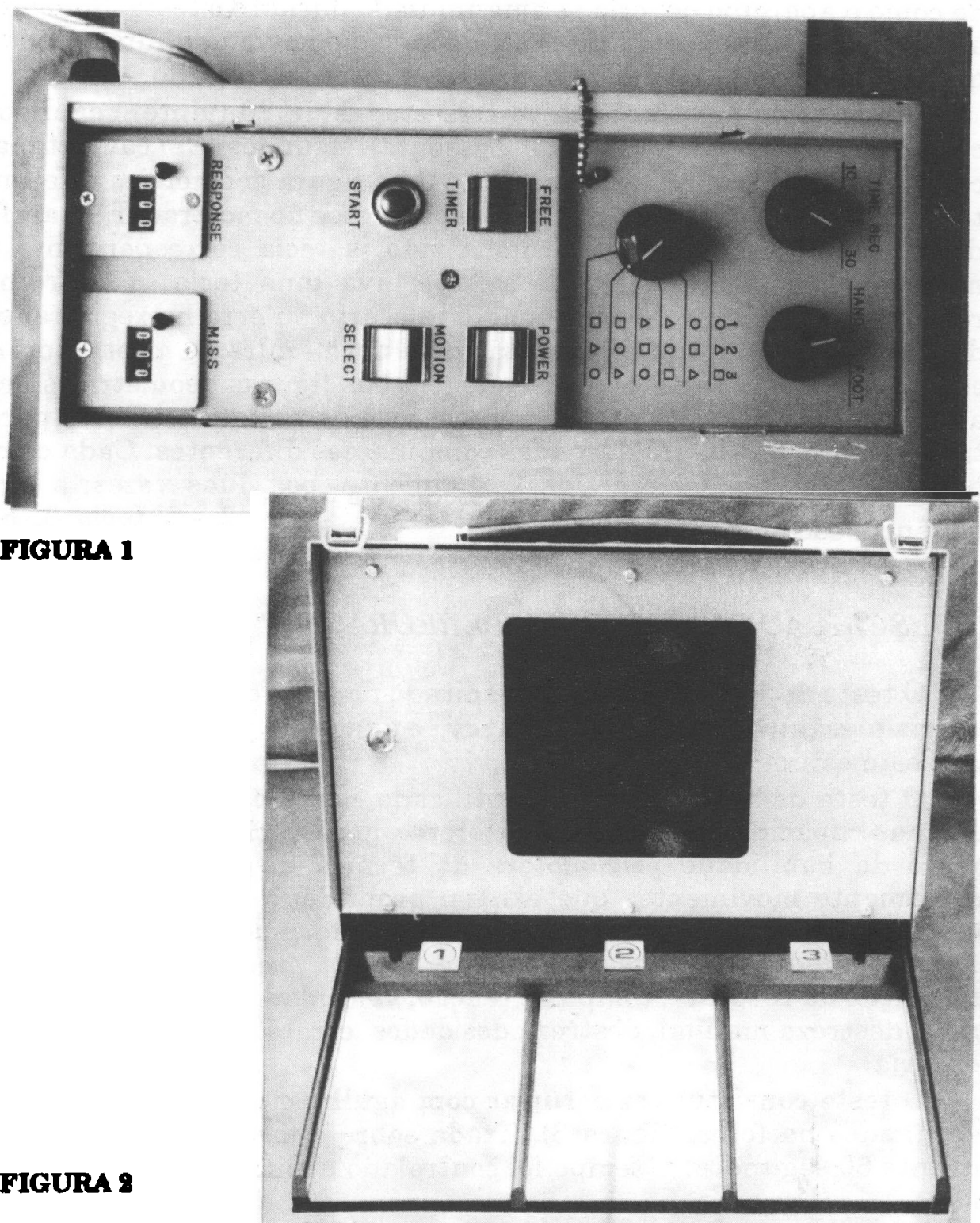


Das capacidades perceptivo-motoras enumeradas por FLEISHMAN $^{8}$, em 1972, as capacidades avaliadas por este teste são: orientação da resposta, tempo de reação, velocidade do movimento do braço, controle de graduação, destreza manual, destreza de dedos, estabilidade braço-mão e velocidade punho-dedos.

Antes da aplicação dos testes, foi esclarecido qual era a finalidade da mesma para o presente estudo, bem como feita uma demonstração de como o aparelho deveria ser manipulado durante o teste. Somente após a verbalização pelo estudante de que não havia nenhuma dúvida quanto ao funcionamento do aparelho, o teste foi iniciado.

Cada teste psicomotor teve três etapas de 20 (vinte) segundos cada, perfazendo um total de 60 (sessenta) segundos. Em cada etapa, cada tecla do aparelho foi associada a uma figura geométrica diferente, de forma que quando uma figura aparecesse no mostrador, a tarefa do estudante seria apertar, com a māo, a tecla correspondente à mesma. No momento em que se apertava uma tecla, o aparelho registrava o ensaio e o correspondente acerto ou erro no registrador de eventos, fazendo aparecer, então uma nova figura no mostrador. A cada etapa de 20 segundos, a seqüência das figuras geométricas era alterada, pois em cada etapa, com exceção do teste psicomotor para treinamento, foram programadas combinaçōes diferentes. Cada combinação diferente foi repetida verbalmente, por duas vezes, a que figura geométrica correspondia a tecla 1 , a tecla 2 e a tecla 3 , na seqüência escolhida.

\section{B. DESCRIÇÃO DO TESTE DE "PERFURAGEM"}

O teste de "perfuragem" foi inspirado no teste de pontilhagem da bateria mecânica de Leon WALTHER ${ }^{29}$ e adaptado pela pesquisadora para este estudo.

O teste de "perfuragem" foi utilizado com o objetivo de avaliar algumas capacidades perceptivo-motoras necessárias ao desenvolvimento da habilidade psicomotora da técnica da IM, que envolve basicamente movimentos que exigem prontidão e precisão motora. Das capacidades perceptivo-motoras enumeradas por FLEISHMAN as capacidades avaliadas por este teste são: precisão de controle, orientaçāo da resposta, tempo de reação, velocidade do movimento do braço, destreza manual, destreza dos dedos, estabilidade braço-mão e pontaria.

O teste consistiu em perfurar com agulha e seringa os círculos desenhados na folha (figura 3), fixada sobre o material de espuma, durante 60 segundos. $O$ tempo foi controlado utilizando-se um cronômetro 


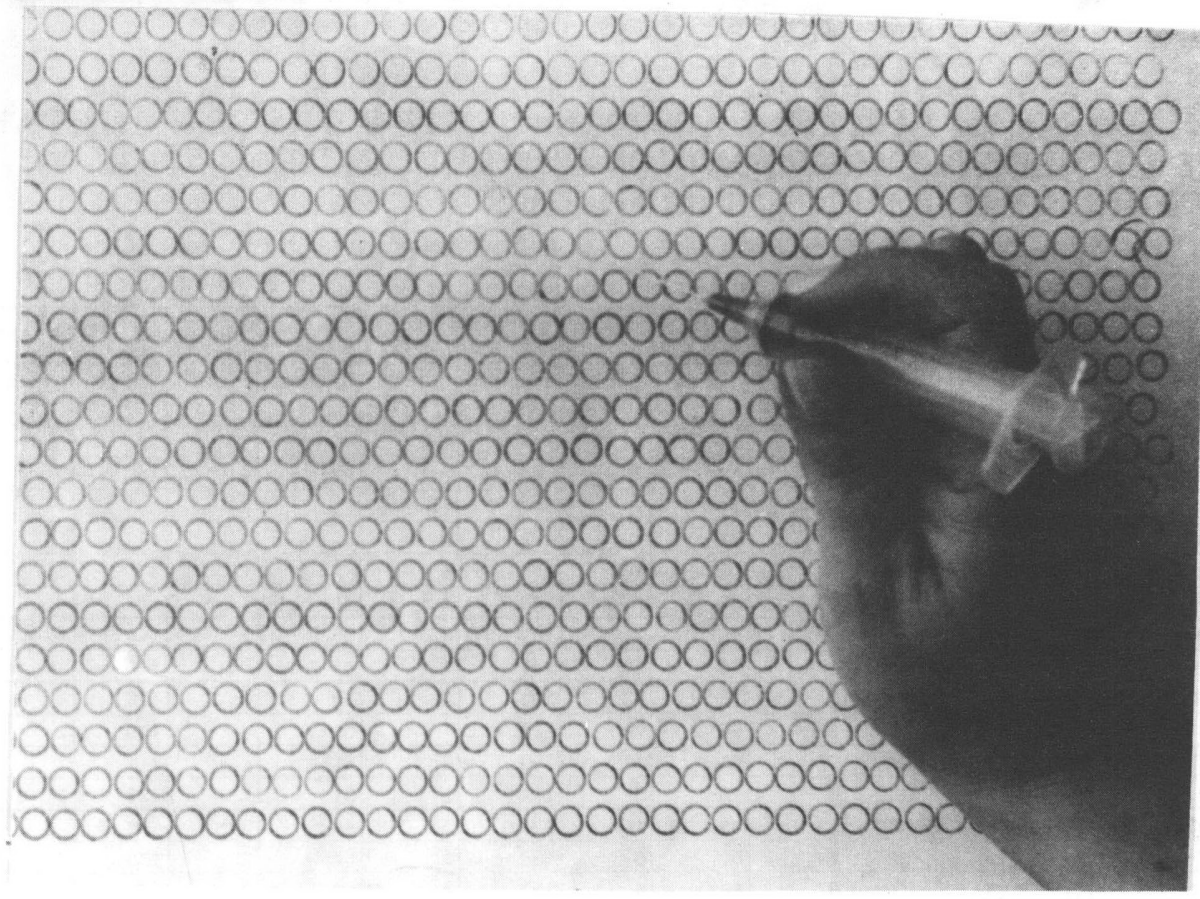

\section{FIGURA 3} tos:

Para a coleta de dados foram elaborados os seguintes instrumen-

- pontuação dos testes instrumentais, utilizados para anotar a pontuaçäo obtida pelo estudante nos testes "psicomotor eletrônico" e de "perfuragem" (anexo I);

- ficha com círculos para o teste de perfuragem, confeccionada em folha de papel sulfite, sem pauta, onde foram desenhados círculos justapostos em linhas horizontais deixando-se bordas laterais de aproximadamente 2 a $2,5 \mathrm{~cm}$ em branco, espaço utilizado para fixaçäo com alfinetes de cabeça, do instrumento de coleta, no material de espuma. Os círculos foram desenhados tendo como base o diámetro do gargalo de uma ampola de $3 \mathrm{ml}$ (aproximadamente o volume que geralmente contém uma medicação de uso intramuscular) (anexo II).

Todos os instrumentos foram previamente testados, antes de serem utilizados no presente estudo.

\section{3 - RESULTADOS}

Os resultados obtidos com a aplicaçāo dos testes instrumentais ("psicomotor eletrônico" e "perfuragem") para avaliação da capacidade psicomotora dos estudantes estāo apresentados no quadro 1 . 
QUADRO 1. PONTUAÇĀO NOS TESTES INSTRUMENTAIS "PSICOMOTOR ELETRÓNICO" E "PERFURAGEM", NOS TRẾS GRUPOS. SÃO PAULO, 1986/1987.

\begin{tabular}{|c|c|c|c|c|c|c|c|c|c|c|c|c|}
\hline \multirow{3}{*}{ 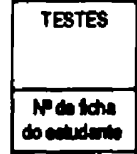 } & \multicolumn{4}{|c|}{ GRUPOA } & \multicolumn{4}{|c|}{ GRUPO B } & \multicolumn{4}{|c|}{ GRUPOC } \\
\hline & \multicolumn{2}{|c|}{ cloomolos } & \multicolumn{2}{|c|}{ perturapen" } & \multicolumn{2}{|c|}{ 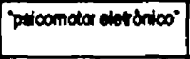 } & \multicolumn{2}{|c|}{ penturagom" } & \multicolumn{2}{|c|}{ 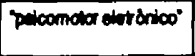 } & \multicolumn{2}{|c|}{ "penturegen" } \\
\hline & conto & -10 & mento & mio & conto & mo & carro & mTo & arto & wro & exto & w10 \\
\hline 1 & $\infty$ & E & 100 & - & 67 & 5 & 124 &. & 58 & 3 & 80 & 8 \\
\hline 2 & 40 & 3 & 114 & 17 & $\infty$ & 2 & 143 & 2 & 81 & 2 & 130 & 7 \\
\hline 3 & 100 & 1 & 144 & 1 & 110 & 7 & 147 & 11 & 73 & 2 & 100 & 1 \\
\hline 4 & 40 & 2 & 102 & 7 & 74 & 1 & 123 & 2 & 51 & 4 & 128 & 18 \\
\hline 5 & 74 & 6 & 148 & 2 & 180 & 16 & 150 & $\theta$ & 42 & 11 & 83 & 5 \\
\hline 6 & $\pi 7$ & 5 & 152 & 13 & 45 & 3 & 148 & 4 & 70 & 1 & 128 & - \\
\hline 7 & 70 & 2 & 149 & 18 & 48 & 4 & 101 & 4 & 55 & 2 & 148 & 4 \\
\hline 8 & 72 & - & 158 & 1 & 99 & 13 & 141 & 2 & 61 & 5 & 140 & 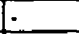 \\
\hline 9 & 48 & 8 & 118 & 7 & 78 & 15 & 135 & 2 & 55 & 3 & 128 & $E$ \\
\hline 10 & 50 & 1 & 125 & 3 & 88 & 7 & 132 & - & 39 & 8 & 100 & 2 \\
\hline 11 & $\infty$ & 1 & 135 & 9 & 65 & 11 & 100 & - & 61 & - & 111 & 1 \\
\hline 12 & 57 & F & 122 & F. & 102 & 6 & 114 & 3 & 81 & 1 & 133 & 3 \\
\hline 13 & 77 & 5 & 163 & 10 & 38 & 11 & 82 & 5 & 46 & 3 & 108 & 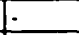 \\
\hline 14 & 81 & 10 & 131 & [e & 51 & 6 & 135 & 2 & 57 & 2 & 135 & 4 \\
\hline 15 & 67 & 4 & 101 & 8 & 80 & 4 & 133 & 4 & 48 & 3 & 190 & - \\
\hline 16 & 50 & 2 & 100 & 5 & 72 & 3 & 127 & 2 & 63 & 1 & 131 & 1 \\
\hline 17 & 82 & 2 & 127 & 1 & 83 & F & 145 & 3 & 51 & 1 & 109 & 5 \\
\hline 18 & 69 & 2 & 112 & - & 75 & 3 & 118 & 1 & 74 & 2 & 139 & 1 \\
\hline 18 & 165 & 5 & 145 & 1 & 67 & 3 & 135 & 3 & 80 & 1 & 134 & 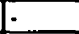 \\
\hline 20 & 65 & 1 & 100 & 4 & 60 & 1 & 112 & 1 & 68 & 2 & 130 & 4 \\
\hline 21 & 81 & 3 & 103 & 7 & 169 & $E$ & 120 & 8 & 93 & 8 & 127 & 4 \\
\hline 22 & 41 & - & 128 & $F$ & 61 & 4 & 108 & 4 & 70 & 3 & 139 & 3 \\
\hline 23 & 50 & 1 & 115 & 1 & 81 & 1 & 120 & 3 & 85 & 9 & 130 & - \\
\hline 24 & 57 & 5 & 86 & 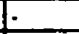 & 83 & 4 & 162 & 3 & 74 & 8 & 118 & 5 \\
\hline 25 & 67 & 5 & \begin{tabular}{|l|l}
132 \\
\end{tabular} & 3 & 108 & 3 & 140 & 2 & 53 & 2 & 120 & 1 \\
\hline 28 & 102 & 2 & 109 & 1. & 72 & $\%$ & 130 & $\%$ & 50 & 1 & 111 & $\%$ \\
\hline 27 & 54 & 1 & 129 & 1 & 57 & 8 & 158 & $\theta$ & 79 & 1 & 128 & 1 \\
\hline 26 & 72 & 1 & 134 & 1 & 05 & 10 & 107 & 8 & 60 & - & 141 & 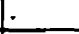 \\
\hline 29 & $\infty$ & 3 & 124 & 2 & 78 & 5 & 117 & 2 & 80 & 1 & 131 & 4 \\
\hline 30 & $\infty$ & 5 & 140 &. & 58 & 8 & 125 & 4 & 804 & 3 & 125 & 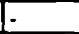 \\
\hline 31 & 70 & 4 & 172 & 3 & 62 & 2 & 74 & 1 & 81 & 1 & 180 & 1 \\
\hline 32 & 160 & 1 & 121 & $1-$ & 102 & 4 & 127 & 1 & 81 & 6 & 135 & 2 \\
\hline 33 & 76 & - & 119 & 1 & 61 & 3 & 137 & 2 & 95 & 9 & 100 & - \\
\hline 34 & 52 & 5 & 120 & 1 & 160 & 5 & 129 & 1 & 82 & 3 & 163 & 3 \\
\hline 35 & 53 & 5 & 133 & 5 & 76 & 5 & 86 & - & 50 & 16 & 75 & 25 \\
\hline 38 & 61 & 5 & 130 & 17 & 70 & - & 117 & 5 & 80 & 3 & 153 & 8 \\
\hline 37 & 60 & - & 88 & 1 & 44 & 6 & 78 & 1 & \begin{tabular}{|l}
97 \\
\end{tabular} & 14 & 144 & - \\
\hline 38 & 75 & 2 & 137 & 1 & 78 & 8 & 149 & 2 & 78 & 2 & 155 & 8 \\
\hline 30 & 71 & 9 & 168 & 3 & 61 & 2 & 114 & 30 & 64 & 3 & 132 & 3 \\
\hline 40 & 43 & 2 & 131 & 1 & 80 & 4 & 181 & 18 & 82 & 2 & 103 & - \\
\hline 41 & 64 & 5 & 104 & 1 & 188 & 4 & 124 & 8 & 61 & - & 108 & 1 \\
\hline 42 & 03 & $\sqrt{2}$ & 134 & 14 & 70 & 4 & 128 & 10 & 91 & 8 & 158 & 5 \\
\hline 43 & 52 & $\%$ & $\infty 8$ & 2 & 69 & $\theta$ & 124 & 18 & 67 & 17 & 128 & 1 \\
\hline 4 & 60 & 4 & 157 & 18 & 103 & 0 & 100 & 5 & 78 & 2 & 117 & - \\
\hline 45 & 57 & 4 & 140 & 5 & 165 & 14 & 111 & 14 & 88 & 16 & 1114 & 8 \\
\hline 40 & 78 & $\sqrt{3}$ & 142 & 18 & 53 &. & 113 & 5 & 106 & 4 & 101 & 4 \\
\hline 47 & 74 & 24 & 133 & 12 & 160 & 5 & 149 & $\%$ & $\infty$ & 10 & 129 & 13 \\
\hline
\end{tabular}


No quadro 1 , na coluna de acertos do grupo $\mathrm{C}$, verifica-se que há duas pontuaçōes discrepantes, correspondendo aos de números 31 e 33. Através da distância de Mahalanobis, a um nível de significância de 2,5\%. (MORRISON ${ }^{22}$ ) esta discrepância foi estatisticamente confirmada. Em vista deste resultado o grupo $\mathrm{C}$ foi redistribuído ficando com um total de 45 estudantes.

A análise de desempenho nos testes instrumentais foi feita apenas com os acertos, devido à uniformidade na proporção de acertos e erros.

Inicialmente foi feita uma análise descritiva, com o uso do programa SYSTAT* 3, calculando-se para cada grupo as medidas de posição, de dispersão e de dependência entre os dois testes.

Assim, os resultados obtidos estāo apresentados nos quadros a seguir.

QUADRO 2. Resultados dos testes instrumentais, nos três grupos. São Paulo, 1986/1987.

\begin{tabular}{|c|c|c|c|c|c|c|}
\hline \multirow[t]{2}{*}{ Resultados } & \multicolumn{2}{|c|}{ GRUPO A } & \multicolumn{2}{|c|}{ GRUPO B } & \multicolumn{2}{|c|}{ GRUPOC } \\
\hline & $\begin{array}{l}\text { "Psicomotor } \\
\text { eletrònico" }\end{array}$ & "Perfuragem" & $\begin{array}{l}\text { "Paicomotor } \\
\text { eletrónico" }\end{array}$ & -Perfuragem" & $\begin{array}{l}\text { "Psicomotor } \\
\text { eletrónico" }\end{array}$ & "Perfuragem" \\
\hline Média & 63,702 & 128.830 & 70,511 & 125,851 & 69.711 & 126,222 \\
\hline Desvio padráo & 10.976 & 20,786 & 15,144 & 21.287 & 15,613 & 18,5461 \\
\hline Erro padráo & 1,601 & $3,032 j$ & 2,209 & 3.105 & 2.3301 & 2,7651 \\
\hline $\begin{array}{l}\text { Coeficuente de } \\
\text { varıaçáo }\end{array}$ & $1) .172$ & 0.161 & 0.215 & 11.169 & 11.224 & 11.1471 \\
\hline Vajor maximo & 83,000 & $|72.000|$ & $102.000 !$ & $|81.000|$ & 105.000 & 163.0001 \\
\hline Valor mınımo & $\$ 1,000$ & 88.000 & 38.000 & $74,000)$ & $39.000 i$ & $75,000) i$ \\
\hline
\end{tabular}

Ainda, para uma melhor visualização da distribuição das variáveis números de acertos nos testes "psicomotor eletrónico" e "perfuragem", foram elaborados os diagramas de dispersảo apresentados nas figuras 4,5 e 6 . 


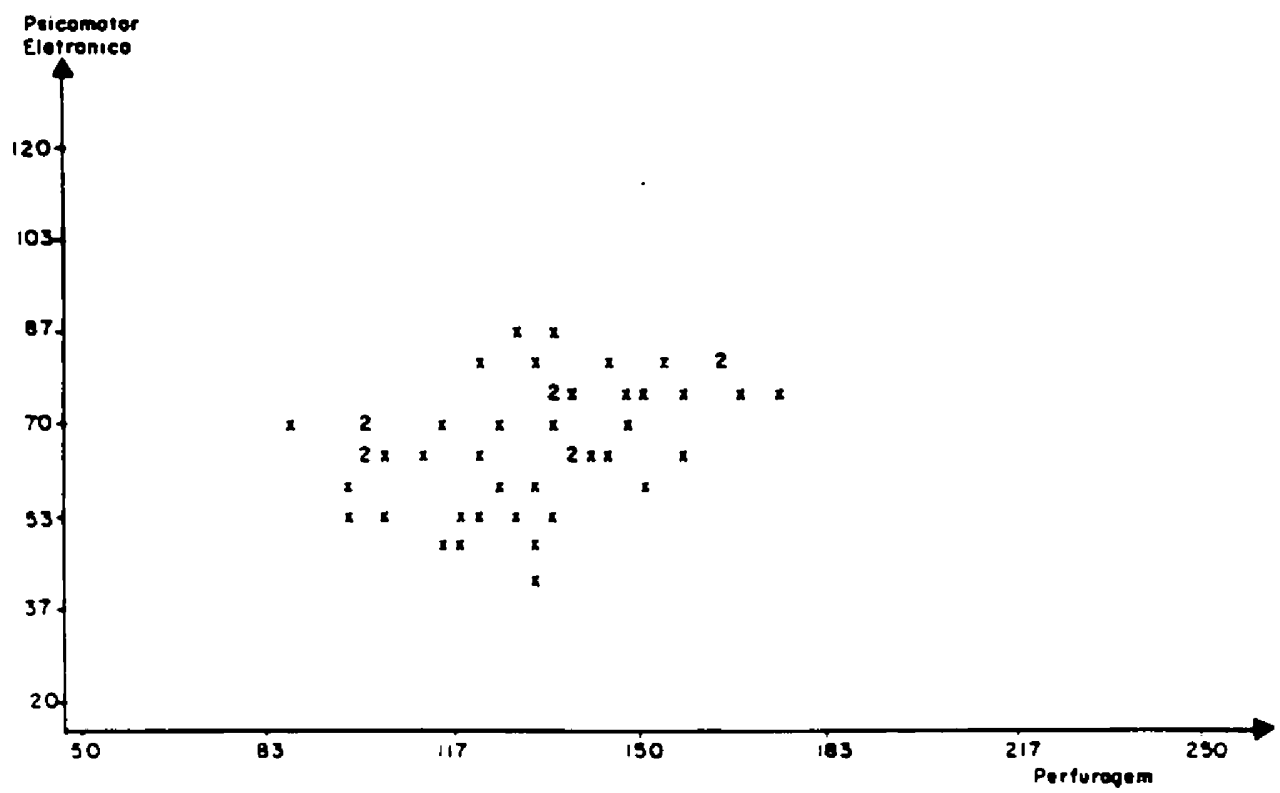

FIGURA 4. Diagrama de dispersaio do número de acertos nos testes instru-

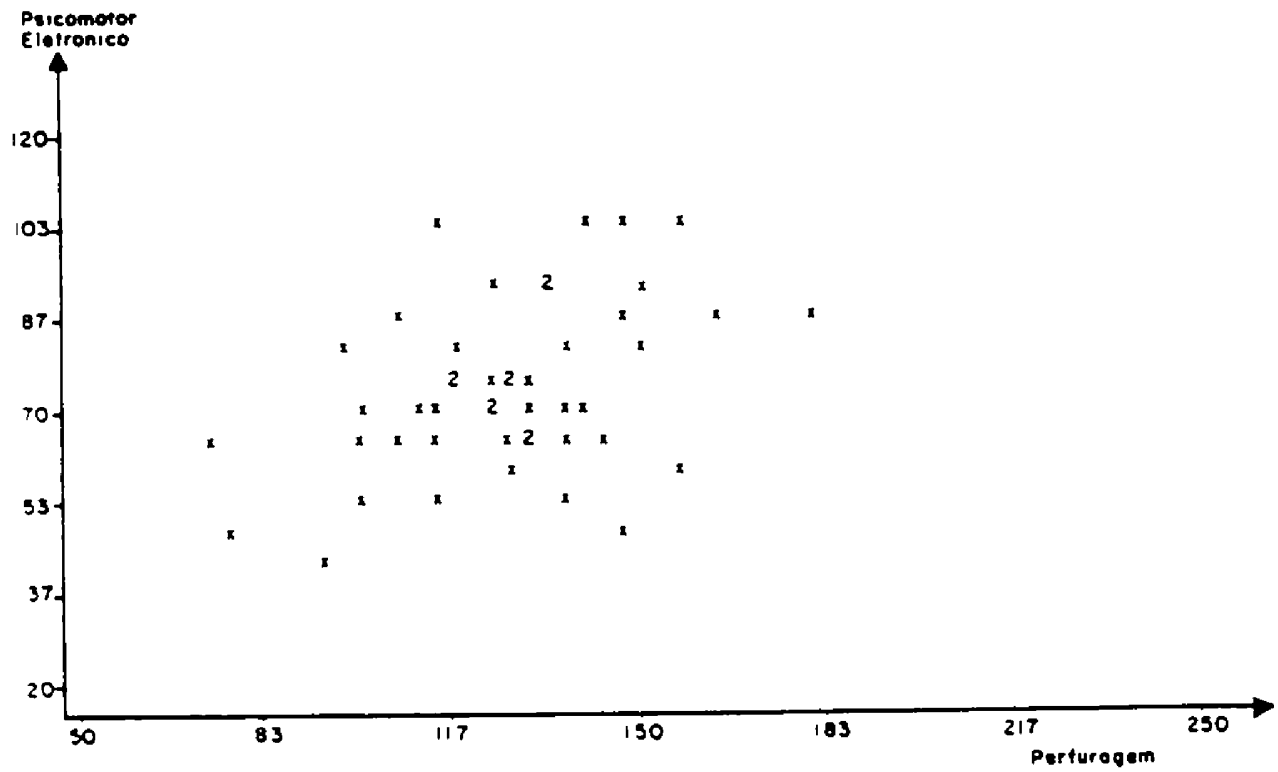

FIGURA 5. Diagrama de dispersào do número de acertos nos testes instru- 


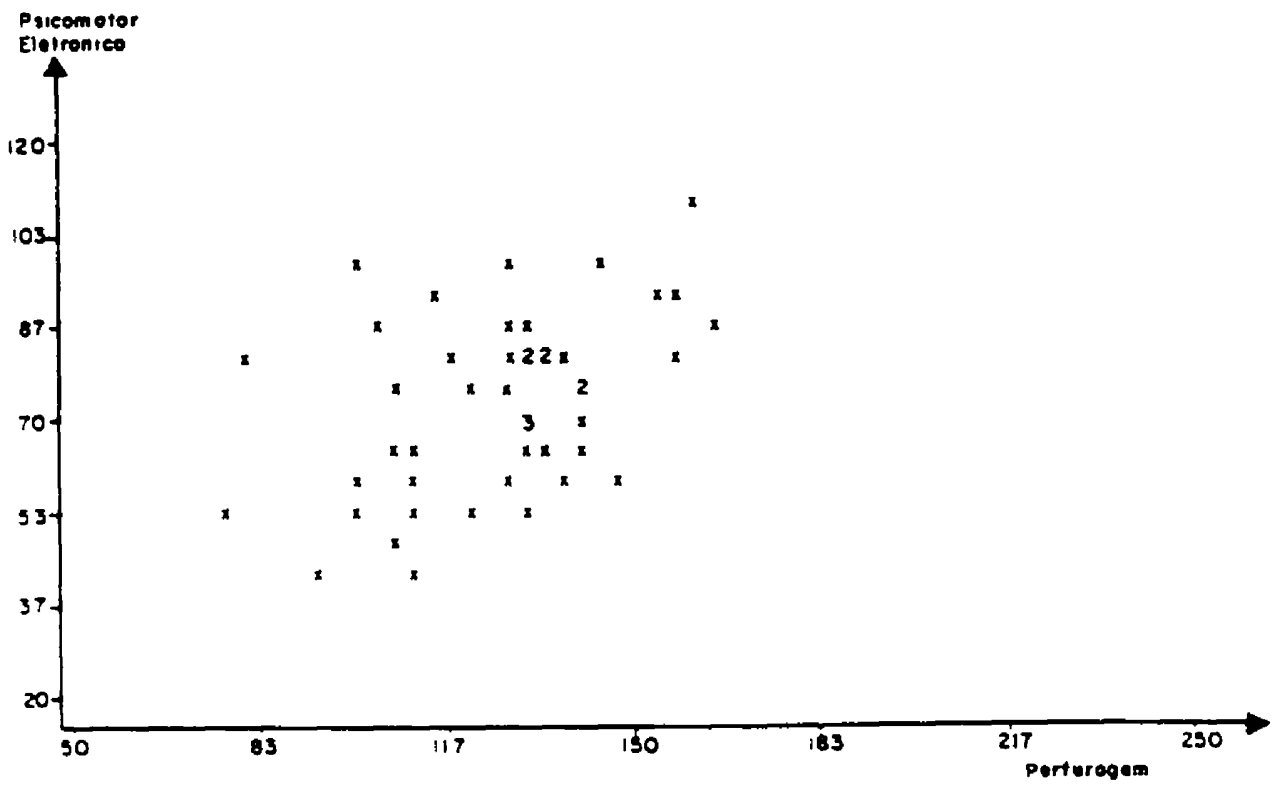

FIGURA 6. Diagrama de dispersào do número de acertos nos testes instru-

Pela análise dos diagramas de dispersäo quanto ao número de acertos nos testes instrumentais, nos três grupos (figuras 4,5,6), concluímos pela nāo existència de argumentos relevantes que contrariem a suposiçăo de normalidade bivariada dos dados em estudo.

Para verificar a correlação entre a variável número de acertos nos dois testes instrumentais, nos três grupos foi calculado o coeficiente de correlação, apresentado no quadro 3.

QUADRO 3. Coeficiente de correlaçāo entre o número de acertos nos dois testes instrumentais, nos três grupos. Sāo Paulo, 1986/1987.

\begin{tabular}{|l|c|c|c|}
\hline GRUPOS & A & B & C \\
\hline $\begin{array}{l}\text { COEFICIENTE DE } \\
\text { CORRELACÄO }\end{array}$ & 0,384 & 0,415 & 0,583 \\
\hline
\end{tabular}

Pelo quadro 3, nota-se que as correlaçōes obtidas são significativas nos três grupos, o que mostra uma relação de dependência acentuada entre os dois testes. Diante deste resultado, realizou-se uma análise da variância bivariada, que testa simultaneamente a igualdade de médias das duas variáveis, ou seja, acertos no teste "psicomotor eletrônico" e acertos no teste de "perfuragem", nos três grupos. 
Para verificar se as matrizes de covariância dos grupos sāo iguais, foi feita uma análise estatística através de uma generalização do teste de Bartlett para QUADRO 4. Teste de igualdade de matrizes de covariância (MORRISON ${ }^{22}$ ).

QUADRO 4. Teste de igualdade de matrizes de covariância. São Paulo, 1986/1987.

\begin{tabular}{|l|r|r|r|}
\hline & $x^{2(1)}$ & G.L. ${ }^{(2)}$ & P-value ${ }^{(3)}$ (aproximado) \\
\hline $\begin{array}{l}\text { Generalização do teste } \\
\text { de Bartlett }\end{array}$ & 9,86 & 6 & 0,130 \\
\hline
\end{tabular}

(1) $\mathrm{x}^{2}$ = quiquadrado

(2) G.L. = grau de liberdade

(3) P-value = nivel descritivo

Para verificar a hipótese de igualdade dos vetores de médias correspondentes aos três grupos, foi utilizada a análise da variância multivariada (JONHSON; WICHERN ${ }^{15}$ )

QUADRO 5. Análise de variância multivariada. São Paulo, 1986/1987.

\begin{tabular}{|l|r|r|r|}
\hline & $F^{(1)}$ & G.L. ${ }^{(2)}$ & P-value ${ }^{(3)}$ (aproximado) \\
\hline $\begin{array}{l}\text { TESTE DE WILK'S } \\
\text { LAMBDA }\end{array}$ & 2,76 & $4 ; 270$ & 0,028 \\
\hline
\end{tabular}

(1) $\mathrm{F}$ = valor estatístico (FISHER)

(2) G.L. = grau de liberdade

(3) P-value = nivel descritivo

Pelos resultados apresentados nos quadros 4 e 5 , pode-se constatar que não há indícios de que as matrizes de covariância, bem como os vetores de médias, sejam diferentes $(P=0,130$ e $P=0,028$ respectivamente).

Assim sendo, a um nivel de significância conjunta de 0,05 , verifica-se que:

os estudantes do $3^{\circ}, 4^{9}$ e $8^{9}$ semestres do curso de Graduaçāo de Enfermagem da USP parecem não diferir quanto à variabilidade do número de acertos no teste "psicomotor eletrônico" e quanto à variabilidade do número de acertos no teste da "perfuragem":

os estudantes do $3^{9}, 4^{q}$ e $8^{q}$ semestres do curso de Graduaçāo em Enfermagem da USP parecem nāo diferir quanto à relaçāo de dependència entre o número de acertos no teste "psicomotor eletrónico" e o número de acertos no teste da "perfuragem"; 
o número médio de acertos no teste "psicomotor eletrônico", conjuntamente com o número médio de acertos no teste da "perfuragem", parece ser o mesmo para os estudantes dos três semestres ( $3^{\circ}$, $4^{9}$ e $\left.8^{9}\right)$.

\section{4 - DISCUSSÃO}

A análise estatística demonstrou homogeneidade entre os três grupos em relação às capacidades psicomotoras testadas.

A média de acertos nos grupos A, B e C (quadro 2) foi respectivamente 63,702; 70,511; 69,711 no teste "psicomotor eletrônico" e de 128,$830 ; 125,851 ; 126,222$ na "perfuragem". A média de erros, nos dois testes, variou entre 3,255 e 5,149 (quadro 1).

A diferença acentuada entre a média dos acertos em relação à média dos erros evidenciou que se tratava de uma população cuja capacidade psicomotora é compatível com a ausència de dificuldades para os fatores testados.

Como já foi mencionado anteriormente, para a avaliaçāo da capacidade psicomotora, procurou-se analisar as condiçōes de coordenaçāo motora, prontidāo motora e memória imediata.

$\mathrm{Na}$ realização dos testes instrumentais pelo estudante, houve predomínio da movimentaçāo voluntária aliada à coordenaçāo motora, prontidāo motora e memória imediata.

Os padrōes da atividade voluntária sāo planejados no cérebro (área pré-motora e motora de córtex)e as ordens sảo enviadas para os músculos através dos sistemas piramidal e extrapiramidal. O sistema piramidal está relacionado com movimentos delicados e de habilidade. O sistema extrapiramidal relaciona-se com movimentos grosseiros e postura (CANELAS; NITRINI ${ }^{3}$ ).

Já, a coordenação motora é essencial para a execução correta de qualquer tarefa. O cérebro e suas conexöes asseguram a devida estabilidade dos mesmos durante a execução da tarefa (CANELAS: NITRINI $^{3}$, ITO $^{14}$, MAGILL $^{16}$, SCHMIDT $^{25}$ ).

Segundo MELARRAGNO FILHO ${ }^{18}$; BRESOLIN ${ }^{18.19}$, as formas mais adequadas e sensíveis de avaliar a memória de um paciente sảo aquelas que avaliam a capacidade para novas aprendizagens. Acreditam que, desta forma, elimina-se a possibilidade de interpretaçōes errôneas, tanto nas respostas que nāo podem ser confirmadas quanto naquelas que dependem do nivel cultural do paciente. A capacidade para aprender coisas novas é um processo de memorizaçāo ativa. que exige maior esforço por parte do paciente do que simplesmente recordar fatos históricos ou pessoais. Sugerem ainda a aplicação de um teste de "repetição de dígitos" capaz de avaliar a memória imediata. 
Este teste consiste na repetição pelo paciente de dígitos após uma leitura (estímulo verbal) ou apresentação impressa (estímulo visual). Inicia-se com uma seqüência de dois dígitos (por exemplo 2 - 5), gradativamente vai se aumentando o número de dígitos para três, quatro, cinco, seis... e assim sucessivamente até o paciente errar. É considerado normal repetir sem maiores dificuldades séries de cinco a sete dígitos. Apresentam, ainda, entre outros testes, o da "retenção verbal", que consiste na reproduçāo verbal, pelo paciente, de uma estória a ele contada através de uma leitura oral. A estória contém 15 itens e um indivíduo normal repete pelo menos 8 itens.

ERICSSON et $a^{6}$, em experimento feito com o objetivo de analisar como a habilidade de memorizar é adquirida, verificaram que um estudante conseguiu expandir sua capacidade de memorizar de 7 a 79 dígitos, após 230 horas de prática no laboratório, utilizando um sistema mnemònico adequado e uma estrutura hierárquica de recuperação. Comprova-se, desta maneira, que, aparentemente, não há limite para expandir a capacidade de memorização atra vés da prática.

Embora não tenha sido possível fazer uma análise dos resultados obtidos confrontando-os com os de outros centros de estudos, julgou-se que se a totalidade dos estudantes obteve acerto em quase todos os dois testes e se a média foi sempre próxima ao valor de acerto de cada estudante, pode-se concluir que as condiçóes de coordenação motora, prontidāo motora e memória imediata foram compatíveis com um bom desempenho da capacidade psicomotora.

Além disso, ficou constatado que as capacidades necessárias ao aprendizado na habilidade de IM estāo presentes de uma maneira homogênea nos très grupos de estudantes, o que permitiria a comparação entre eles.

Outrossim, julga-se que estes testes ou outros de natureza similar poderiam ser utilizados para determinar a capacidade do estudante em relaçāo ao aprendizado de habilidades psicomotoras complexas.

\section{5 - CONCLUSĀO}

Neste estudo os três grupos de estudantes (A, B e C) demonstraram ser homogêneos em relaçáo às capacidades psicomotoras. avaliadas através dos testes instrumentais, "psicomotor eletrónico"e "perfuragem".

MTYADAHIRA. A. M. K.; KOIZUMI. M. S. Measurement of psychomotor ability to the performance of intramuscular procedure. Rev. Esc. Enf. USP., v. 27, n.2, p. 263-80, aug. 1993. 
This study is to verify the psychomotor skill using psychomotor capacity measure. The authors describes two tests that will be used by nurses denominated "eletronic psychomotor" and "perfuration". Those tests are applied in the first stage of this study to analyse the teaching-learning process of intramuscular injection. Three groups of undergraduate nursing student in difference stages of evolution of the course, were analysed and were verified that there was homogeneity among groups in relation to psychomotor capacity.

UNITERMS: Infection intramuscular. Ability poycomotor

\section{REFERÊNCIAS BIBLIOGRÁFICAS}

1. ALMEIDA, M.C.P. Estudo do saber de enfermagem e sua dimensào prática. Rio de Janeiro, 1984. 179p. Teser Doutorado)-Escola Nacional de Saúde Pública, Fundaçáo Oowaldo Cruz.

2. BUTTERFIELD, S.E. In defence of the demonstration room. IntNurs.Rev., v.30, n.1. p.15-20, 1983.

3. CANELAS, H.M.; NITRINI, R. Controle segmentar da motricidade. In: ASSIS, J.L. de; SCAFF, M. Fisiopatologia do sistema nervoso. São Paulo, Sarvier, 1983. cap.12, p.49-64.

4. CARVALHO, V.L. Ensino de enfermagem e metodologia. 2.ed. Rio de Janeiro, Cultura Médica, 1979.

5. ELLIOT, R. et al. Psychomotor skill acquisition in nuraing students in Canada and the U.S.A. Can.Nurse, v.78, n.3, p.25-7, 1982.

6. ERICSSON, K.A. et al. Acquisition of a memory skill. Science, v.208. n.6, p.1181.2, 1980.

7. FLEISHMAN, E.A. Structure and measurement of physical fitness. New York, Prentice-Hall, 1964. cap.2, p.8-27. The psychomotor donain. Philadelphia, Lea \& Febriger, 1972. p.78.106.

9. FRIEDLANDER, M.R et al. Avaliaçào das habilidades psicomotoras em enfermagem: subsidios para a construçáo de um instrumento. Rev.Paul.Enf., v.4, n.2, p.72-7, 1984 .

10. GOMEZ, G.E.; GOMEZ, E.A. The teaching of psychomotor skill in nursing. Nurse Educ., v.9, n.4, p. 35.9, 1984

11. HARDY, L.K. Keeping up with "Mrs.Chase": an analysis of nursing skill.learning. J.Adv.Nurs.. v. 5, n.3, p.321.7, 1980.

12. HORTA. W A. et al. O ensino dos instrumentos básicos de enfermagem. Rev.Esc.Enf.USP, v.4, n.1/2, p.5.20, 1970.

13. INFANTE, M.S. Toward effective and efficient use of the clinical laboratory. Nurse Educ., v. 6, n.1, p. 16-9, 1981.

14. ITO, M. Neurophysiological aspects of the cerebellar motor control system. Int.J.Neurol., v. 7, p.162-76, 1970

15. JOHNSON, R.A. WICHERN, D.W Applicaed multivariate statigtical analysis. Englewood Cliffs. Prentice Hall, 1982.

16. MAGILl. R.A. Aprendizagem motora: conceitos e aplicaçóes. Sảo Paulo, Edgard Blucher. 1984. Cap. 1, p. 2-23: O dominio motor. 
17. MAGIIL, R.A. Aprendizagem motora: conceitos e aplicaçōes. Sāo Paulo, Edgard Blucher, 1984. Cap.7, p. 156-69: Diferenças individuais.

18. MELARAGNO FILHO, R.; BRESOLNN, A.V. Maneira prtitica de avaliaçāo da membrias parte 2. Sáo Paulo, Laboratório ACHE, s.d., p. 15-27. (a)

19. MELARAGNO FILHO, R.; BRESOLIN, A.V. Mnneira prtitica de avaliação da memórias parte 3. São Paulo, Laboratório ACHE, s.d., P. 31-9. (b)

20. MILDE, F.K. The function of feedback in poychomotor skill learning. Weat.J.Nurs.Res., v.1, n.4, p. 425-34, 1988. 


\section{TESTE "PSICOMOTOR ELETRÔNICO"}

Tempo de cada etapa (20 segundos) - Total das três etapas (60 segundos).

\begin{tabular}{|l|l|l|l|l|l|l|l|}
\hline RESULTADO & $\begin{array}{c}\text { seqüência/ } \\
\text { etapa }\end{array}$ & \multicolumn{3}{|c|}{ IGUAL } & \multicolumn{3}{c|}{ DESIGUAL } \\
\hline & & I & II & III & I & II & III \\
\hline scerto & & & & & & & \\
\hline erro & & & & & & & \\
\hline Total & & & & & & & \\
\hline Pontuação & & & & & & & \\
\hline
\end{tabular}

TESTE DE “PERFURAGEM"

Tempo (60 segundos)

\begin{tabular}{|l|l|l|}
\hline \multicolumn{1}{|c|}{ RESULTADO } & Perfuração & Número \\
\hline acerto & & \\
\hline erro & & \\
\hline TOTAL & & \\
\hline PONTUAÇĀO & & \\
\hline
\end{tabular}

Observaçōes: 


\section{ANEXO II}

$8.80080000008808988 \%$

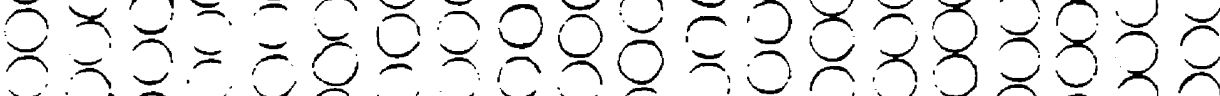

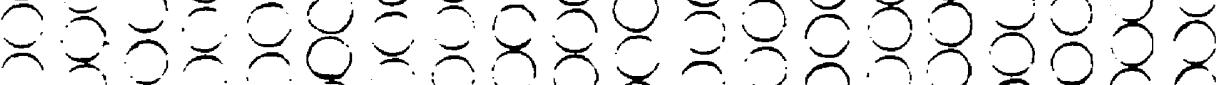

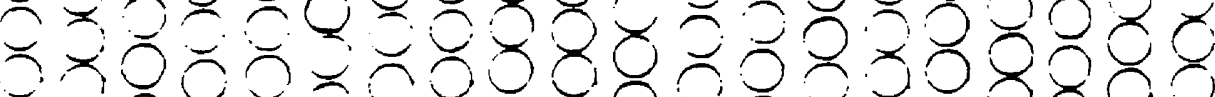

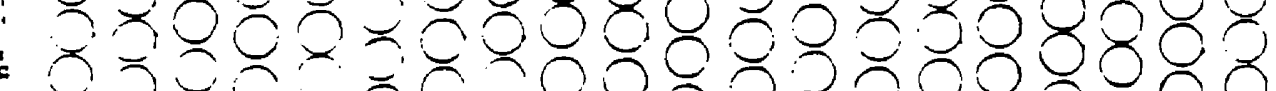
辰

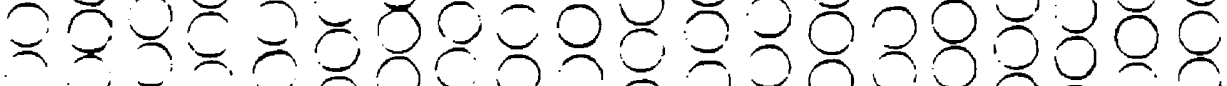

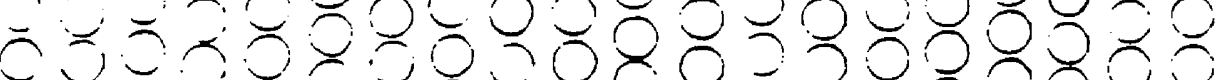

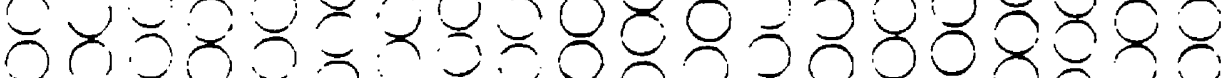

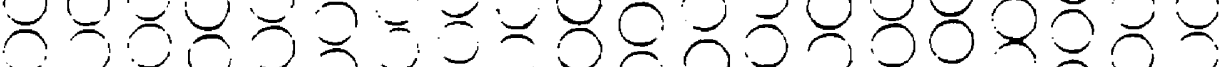

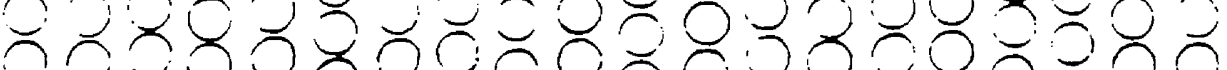
×

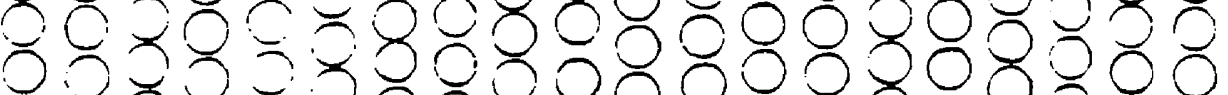
$0.50=08080008080080$ $0 \times 0.0 .0 \times 00800=080 \%$ ○00 00000800008000 二 00000000000008088

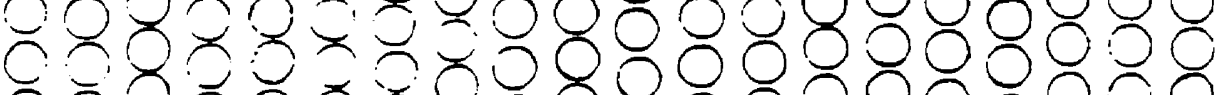
\%7\%

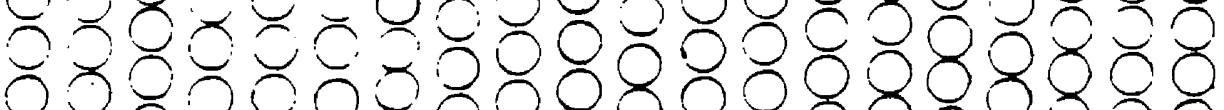

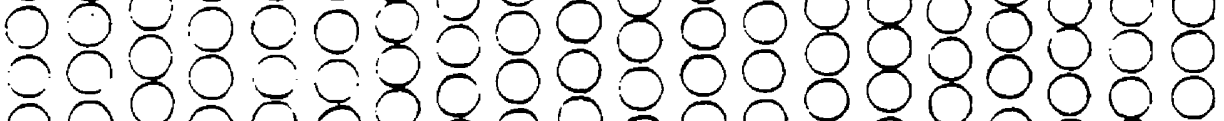

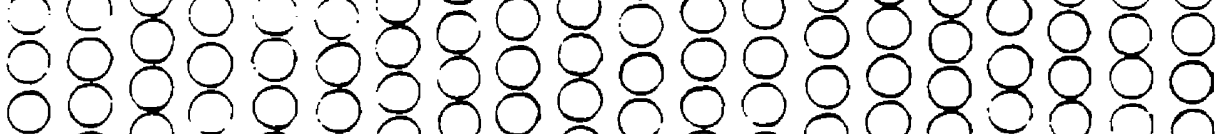
$088,0.0000000008080$ 08080880808008080080 0080088000008888808 $\circ 080000000080808$ $\circ 080000008080808$ 08808808088080808080 0080008080000080008 08808088808088080080 